\title{
An integrated LCA/LCC framework for assessing product sustainability risk
}

\author{
N. Palousis, L. Luong \& K. Abhary \\ School of Advanced Manufacturing \& Mechanical Engineering, \\ University of South Australia, Australia
}

\begin{abstract}
Design for Environment is a design approach used to improve the performance of products and manufacturing operations, and reduce business disruption and sustainability risk, through design characteristics that reduce environmental impacts over the product's entire life cycle. For a manufacturing company, 'sustainability risk' includes those risks resulting from the emerging environmental and social justice issues (such as greenhouse emissions and waste legislation) that directly affect the quality, cost and development time of a product line. Identifying and assessing these risks at the product design stage is crucial for the development of products that both minimise sustainability risk exposure and are environmentally sustainable. Sustainability Risk Assessment at the product design stage must link the environmental impact of a product to business risks that affect the product life cycle cost. This paper describes a framework that integrates Life Cycle Analysis and Activity-Based Life Cycle Costing with risk assessment to identify, assess and model the impact of sustainability risks specific to a product's life cycle characteristics.
\end{abstract}

Keywords: sustainability, risk assessment, Life Cycle Costing, Life Cycle Analysis, Sustainability Target Method, climate change, Design for Environment.

\section{Introduction}

The global issues of climate change, resource consumption and waste are translating into real business risks and opportunities that are forcing Australian manufacturers to design their products more sustainably. Particularly, the Australian Government's renewed focus on climate change response through 
ratification of the Kyoto Protocol, passing of the National Greenhouse and Energy Reporting Act, and introduction of a National Emissions Trading Scheme by 2010 will have energy, transport and materials cost implications for the manufacturing sector (Ernst \& Young 2007 [1]). Furthermore, the increasing presence of product stewardship regulations, such as the Waste Electrical and Electronics Equipment (WEEE) EC 2002 [2] and End of Vehicle Life (ELV) EC 2000 [3] directives are placing pressure on manufacturers to rethink entire product and service lines, and other means for ensuring operational costs remain bearable in a carbon- and resource-constrained environment.

For a typical product, $70 \%$ of the cost and environmental impact of development, manufacture and use is determined in its design phase (Yarwood and Egan [4]). Therefore, in response to the emerging demand by companies for sustainable business practices, the manufacturing industry is adopting product development approaches such as Design for Environment to reduce business disruption and sustainability risk, through design characteristics that reduce environmental impacts over the product's entire life cycle.

\section{Review of literature}

\subsection{Defining sustainability risk}

The International Standardisation Organisation (ISO) [5] defines industry 'risk' as "the combination of the probability of an event and its consequences". A manufacturing company can face business risks resulting from internal factors, such as the supply chain (operational risks), or external factors, such as regulations (regulatory risk) (Institute of Risk Management [6]).

Risk by nature is complex and interrelated, requiring a holistic, systemic and multidisciplinary approach to be successfully managed. To understand the impact of complex and interrelated issues of climate change and sustainability on manufacturing business, a risk assessment approach is required for the development of sustainable products and services (Haimes [7], Andrews and Moss [8], Anderson [9], Williams [10]).

Anderson [11] defines 'sustainability risk' to define those business risks (i.e. that impact on economic cost or value) resulting from the environmental and social justice challenges faced by business, such as: global warming/climate change, diminishing water supplies and contamination, diminishing ecosystems services, gender/racial discrimination, unfair workplace practices and corruption in developing countries. Specific to climate change, the Carbon Disclosure Project [12] and SAM [13] identify some broad categories of sustainability risk to businesses, which translate into particular types of risk that directly impinge a manufacturing company, summarised in table 1 .

The literature reviewed highlights two common criteria to characterise sustainability risk:

1. Each risk is directly or indirectly attributable to a form of environmental impact or event, and

2. Each risk has a direct or indirect impact on the life cycle cost or value of a product, service, project or entire organisation. 
Table 1: General categories of climate change business risk (adapted $[12,13])$.

\begin{tabular}{|l|l|}
\hline Risk Category & \multicolumn{1}{|c|}{ Description } \\
\hline Physical risks & $\begin{array}{l}\text { The physical impacts of climate change e.g. the } \\
\text { direct impact of rising sea levels, droughts, floods, } \\
\text { and storms on direct manufacturing operations }\end{array}$ \\
\hline Regulatory risks & $\begin{array}{l}\text { The regulatory costs and impacts e.g. when } \\
\text { manufacturers must pay to emit or off-set emissions } \\
\text { via trading schemes, or situations where companies } \\
\text { must meet the cost of regulatory compliance }\end{array}$ \\
\hline Litigation risks & $\begin{array}{l}\text { Companies may face legal action if they fail to meet } \\
\text { legal obligations associated with climate change- } \\
\text { related issues e.g. Kyoto Protocol requirements }\end{array}$ \\
\hline Competitive risks & $\begin{array}{l}\text { Companies that fail to take proactive steps to } \\
\text { mitigate climate change-related risk may lose a } \\
\text { competitive advantage in the medium to long term }\end{array}$ \\
\hline Reputational risks & $\begin{array}{l}\text { Companies may be exposed to consumer backlash if } \\
\text { their reputation is damaged because of their poor } \\
\text { responsiveness }\end{array}$ \\
\hline Supply chain risks & $\begin{array}{l}\text { The disruptive or cost impacts on the supply chain } \\
\text { e.g. the cost of materials and transport increases } \\
\text { with fuel prices and emissions regulations }\end{array}$ \\
\hline
\end{tabular}

\subsection{Sustainability Risk Assessment at the product level}

Managing sustainability risk for a company at the product level is thus a function of both a product's environmental impact and its economic cost; to manage the impact of sustainability risk on the life cycle cost of a product, the life cycle environmental impact of the product must be assessed to determine what types of sustainability risk are relevant, the probability that they will occur and the severity of impact on the product life cycle cost.

Therefore, the use and potential integration of existing design methodologies that assess environmental impact and cost impact across the product's life cycle is a fundamental requirement of product-level Sustainability Risk Assessment. While DfE does provide a broad philosophy to minimising the business risks associated with sustainability at the product level, functional methodologies are required for the evaluation of environmental impacts and risks, in relation to the costs and benefits of corrective interventions (Reis et al, 1999 [14], Anderson [11]). Existing best-practice functional methodologies in the literature either solely assessing environmental impact (Life Cycle Assessment (LCA)) (ISO [15]), or life cycle cost (Activity Based-Life Cycle Costing (AB-LCC)) (Embelmsvåg [16]). In order to wholly assess sustainability risk, these tools must be integrated. A framework and methodology is therefore needed that integrates LCA and LCC, and enables the assessment and modelling of sustainability risk specific to a product's life cycle characteristics. 


\section{Sustainability Risk Assessment framework}

\subsection{Framework overview}

The Sustainability Risk Assessment framework integrates two existing and wellestablished methodologies, commonly used at the design stage of the product development process, to determine both the environmental impact and economic cost for the entire product life cycle - Life Cycle Assessment and Activity Based Life Cycle Costing (fig 1).

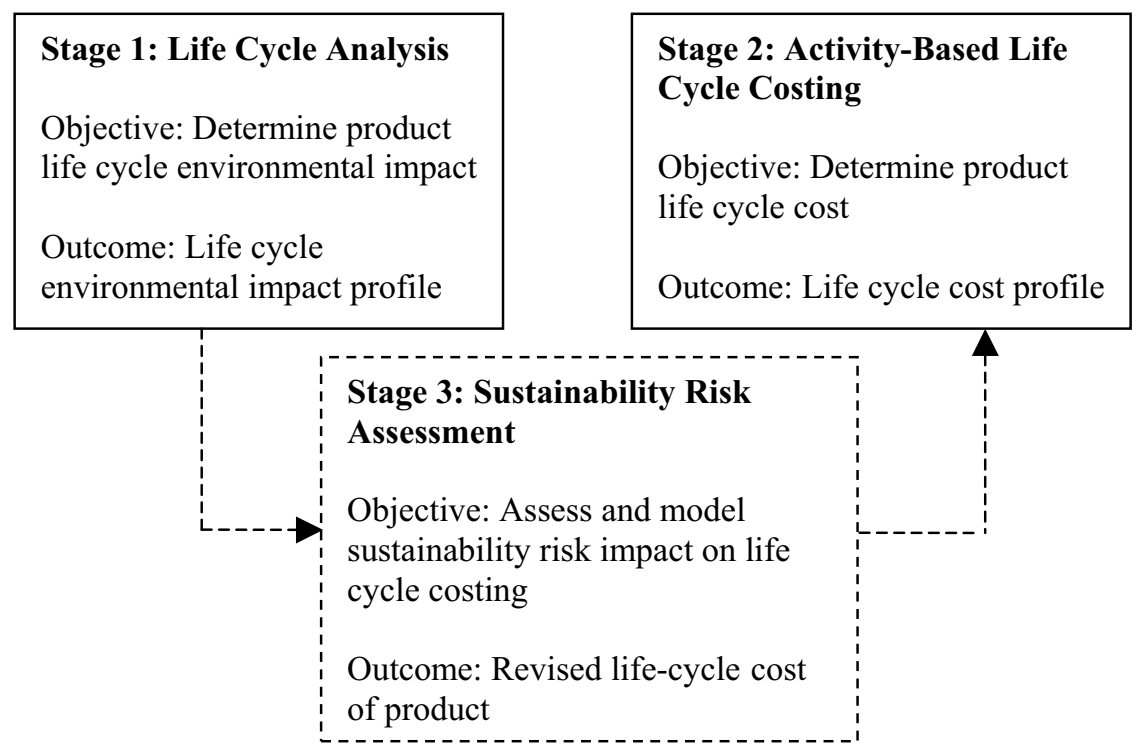

Figure 1: $\quad$ Sustainability Risk Assessment framework.

Stage 1 conducts a LCA of the product to determine the level of environmental impact from the consumption of materials and activities throughout the product life cycle. The LCA utilises the Sustainability Target Method (STM) to determine the degree of 'eco-efficiency' $(E E)$ at each stage of the product life cycle (Swarr et al [17]), as an indicator for determining the type and probability of sustainability risks attributable to the environmental impact profile. Stage 1 involves the three functional steps of LCA: define scope of analysis, create life cycle inventory, and conduct a life cycle impact assessment ISO [15].

Stage 2 involves applying the Activity-Based Life Cycle Costing methodology to assess the life cycle cost of the product, based on the consumption of materials, resources and activities. The methodology is guided by a 10-step modelling and calculation process, which, depending on the resulting data, can be undertaken recursively to obtain greater accuracy or suitability (Emblemsvåg [16]). 
Stage 3 applies the Sustainability Risk Assessment methodology to identify and assess sustainability risks across the product life cycle, and model the impact of sustainability risk to the product's life cycle cost profile. The methodology integrates the LCA eco-efficiency results from Stage 1 with fuzzy logic to assess the probability and severity of identified sustainability risks to the product life cycle, and feeds the resulting risk impact factors to the Activity-Based LCC to provide a risk-adjusted life cycle cost profile. Stage 3 comprises of 5 risk identification and assessment steps, and a final step of modelling the sustainability risks (Monte Carlo analysis) and revising life cycle costing data.

\subsection{Example application of Sustainability Risk Assessment: a toy car}

Consider the following basic example of the SRA framework to product manufacturing. An Australian manufacturer of children's toys is considering upgrading its existing 'cradle-to-grave' manufacturing system for a specific toy car product line and implementing a new product take-back and reuse/recycle system. Initial life cycle costing revealed the new system to be too expensive to implement, relative to the existing system. However, recognising the future risks and financial implications associated with waste regulations, petrol prices and greenhouse emissions, a revised costing was requested to take into account the potential impacts of sustainability risks. The results are depicted in Table 2, and the SRA procedure outlined below (excludes detailed analyses and modelling).

Stage 1: Life Cycle Analysis. A systems model is built of the environmental input/output flows describing the proposed cradle-to-cradle manufacturing system. Life Cycle Inventory data (amount of resource use and pollutant emissions per functional unit) is collected across the life cycle components of 'Supply Chain', 'Manufacturing', 'Use' and 'Reuse/Recycle/Disposal'. Life Cycle Impact Assessment is undertaken with the Sustainability Target Method to calculate the $(E E)$ value for each of the product life cycle components.

Stage 2: Activity-Based Life Cycle Costing. The procedure uses Activity Based Costing, activity networks and Monte Carlo analyses to estimate and model the toy car's bill of activities, and hence total cost across the product life cycle.

Stage 3: Sustainability Risk Assessment (SRA). The SRA procedure involves identifying sustainability risks specific to the toy car life cycle, mapping (using causal loop networks) each sustainability risk to one or more stages of the toy car product life cycle, and applying fuzzy logic to assess the probability and severity of each sustainability risk on the product life cycle. The assessment process uses the $E E$ values obtained in Stage 1 to govern the probability $(P)$ of a sustainability risk; the severity of the sustainability risk $(S)$ is considered the percentage increase in cost that a life cycle activity will occur if that risk occurred. Finally, Steps 7-10 of Stage 2 are revised to incorporate the potential impact of defined sustainability risks on the life cycle cost of the toy car.

The results show that, once the potential sustainability risks are factored in to the life cycle costing of both the existing and new systems, the new system is now economically feasible for implementation. It is observed that the existing 


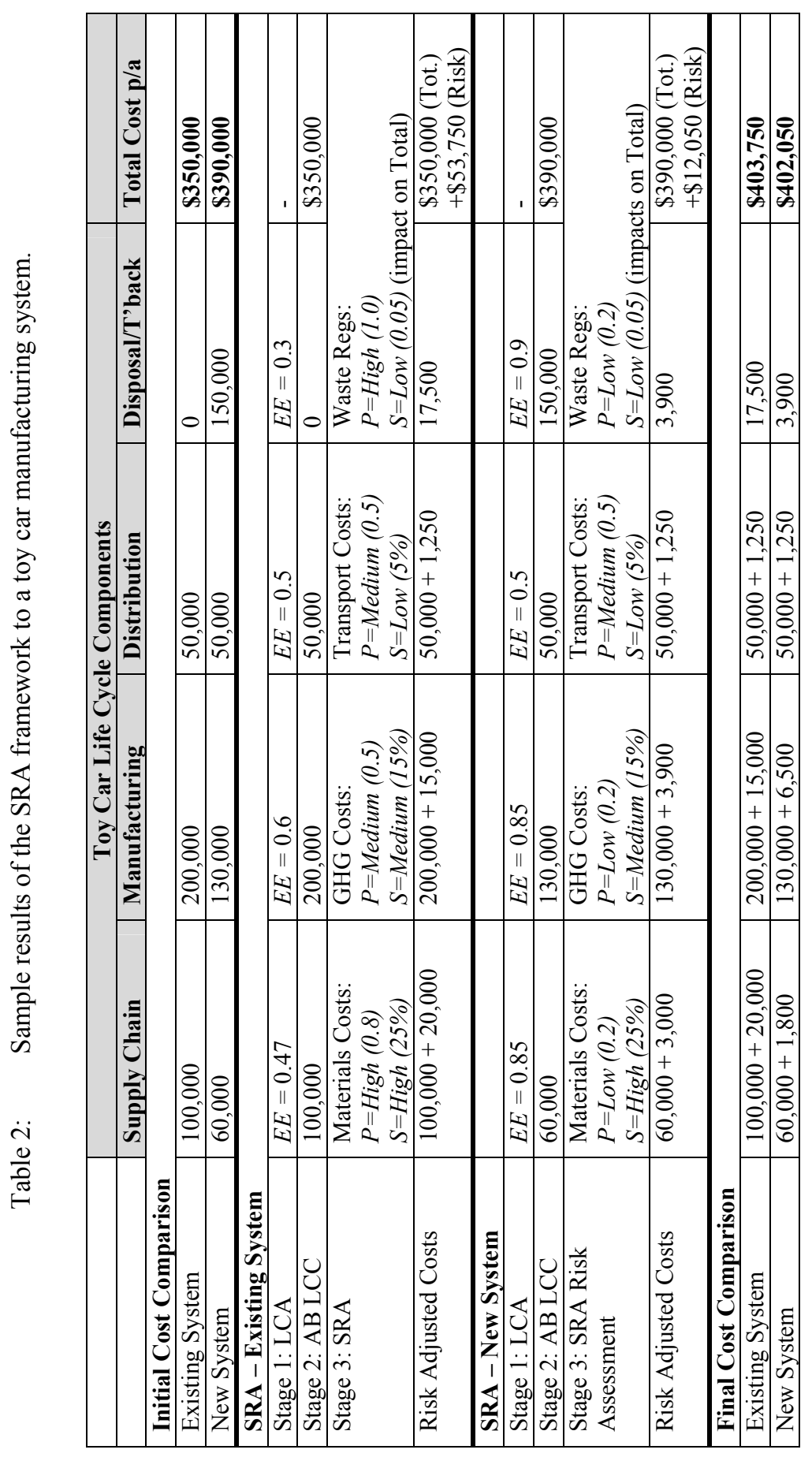


system is more susceptible to cost increases that the new system. This is primarily due to the new system using fewer materials (by feeding recycled materials back through to manufacturing), less manufacturing costs (as parts are being reused and therefore don't need to be manufactured), and less exposure to the fines and costs associated with waste and product take back regulations. (Note that this example only considers single risks for each stage of the product life cycle; in reality the problem is much more complex, with multiple and interrelated risks within and across life cycle components. The modelling component of the SRA is useful in identifying the impacts of multiple, interrelated sustainability risks.)

\section{Conclusion}

This paper outlines a framework that integrates Life Cycle Analysis and Activity-Based Life Cycle Costing with risk assessment to identify, assess and model the impact of sustainability risk on the product life cycle. This framework is used at the early design stage of product development to guide the process of materials and process selection for minimising sustainability risk. More work is being done on the development of the Sustainability Risk Assessment framework and its application to the automotive sector. In partnership with a multinational automotive components manufacturer, the authors are applying the Sustainability Risk Assessment framework to case studies of electronic mirror actuator manufacturing.

\section{References}

[1] Ernst \& Young, The Rudd government's commitment to combating climate change, Ernst \& Young: Sydney, 2007.

[2] European Commission (EC), Waste Electrical \& Electronics Equipment (WEEE) 2002/96/EC, EC: Brussels, 2002.

[3] European Commission (EC), End of Vehicle Life Directive (ELV) 2000/53/EC, EC: Brussels, 2000.

[4] Yarwood, J.M. and Eagan, P.D. Design for the Environment Toolkit: A Competitive Edge for the Future, Minnesota Office of Environmental Assistance: Minnesota, 1998.

[5] International Organisation for Standardization (ISO), ISO/IEC Guide 73:Risk management -- Vocabulary -- Guidelines for use in standards, ISO, Geneva, Switzerland, 2002.

[6] Institute of Risk Management (IRM) A Risk Management Standard, IRM: London, UK, 2002.

[7] Haimes, Y. Risk Modelling, Assessment and Management, John Wiley \& Sons: New Jersey, pp. 33-35, 2004.

[8] Andrews, J.D. and Moss, T.R. Reliability and Risk Assessment (2 ${ }^{\text {nd }}$ Edition), American Society of Mechanical Engineering, 2002.

[9] Anderson, T.J. Perspectives on Strategic Risk Management, Copenhagen Business School Press: Herdnon, VA, 2002. 
[10] Williams, T. M. (2000) Systemic project risk management - the way ahead. International Journal of Risk Assessment and Management, Inderscience: Onley Bucks, UK, Vol. 1, Nos. 1/2, 2000.

[11] Anderson, D.R. Sustainability Risk Management. CPCU eJournal, Chartered Property Casualty Underwriters: Malvern, PA, May 2006.

[12] Carbon Disclosure Project Carbon Disclosure Project Report 2006: FT 500, Innovest: New York, 2006.

[13] Sustainable Asset Management (SAM) \& World Resources Institute (WRI) Changing Drivers: The Impact of Climate Change on Competitiveness and Value Creation in the Automotive Industry, SAM: Zurich, n.d.

[14] Ries, G. Winkler, R. and Zust, R. Barriers for a successful integration of environmental aspects in product design. Proc. of EcoDesign '99: $1^{\text {st }}$ International Symposium on Environmentally Conscious Design and Inverse Manufacturing, IEEE: Tokyo, pp527-532, 1999.

[15] International Organisation for Standardisation (ISO) ISO 14040: 2006 Environmental Management - Life Cycle Assessment - Principles and Framework, ISO: Geneva, 2006.

[16] Emblemsvåg, J. Life-Cycle Costing. Using Activity-Based Costing and Monte Carlo methods to manage future costs and risks, John Wiley \& Sons: New Jersey, 2003.

[17] Swarr, T.E. Cline, H.J. Jeong, S. Dickinson, D.A. and Caudill, R.J. Evaluating Supply Line Sustainability and Business Environmental Risk, IEEE International Symposium on Electronics and the Environment, 2004. 